Supporting Information

\title{
Electrochemically sensing of trichloroacetic acid with iron(II) phthalocyanine and Zn-based metal organic framework nanocomposites
}

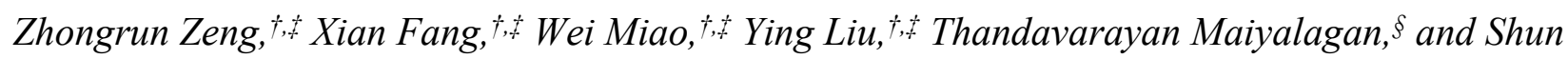

$$
\mathrm{MaO}^{*}+\dot{\dagger}, \dot{t}
$$

$\dagger$ Biomedical Multidisciplinary Innovation Research Institute, Shanghai East Hospital, State Key Laboratory of Pollution Control and Resource Reuse, College of Environmental Science and Engineering, Tongji University, 1239 Siping Road, Shanghai 200092, P.R. China

$\$$ Shanghai Institute of Pollution Control and Ecological Security, Shanghai 200092, P.R. China

$\S$ Electrochemical Energy Laboratory, Department of Chemistry, SRM Institute of Science and Technology, Kattankulathur 603203, India

*Corresponding author: shunmao@tongji.edu.cn (S. Mao) 

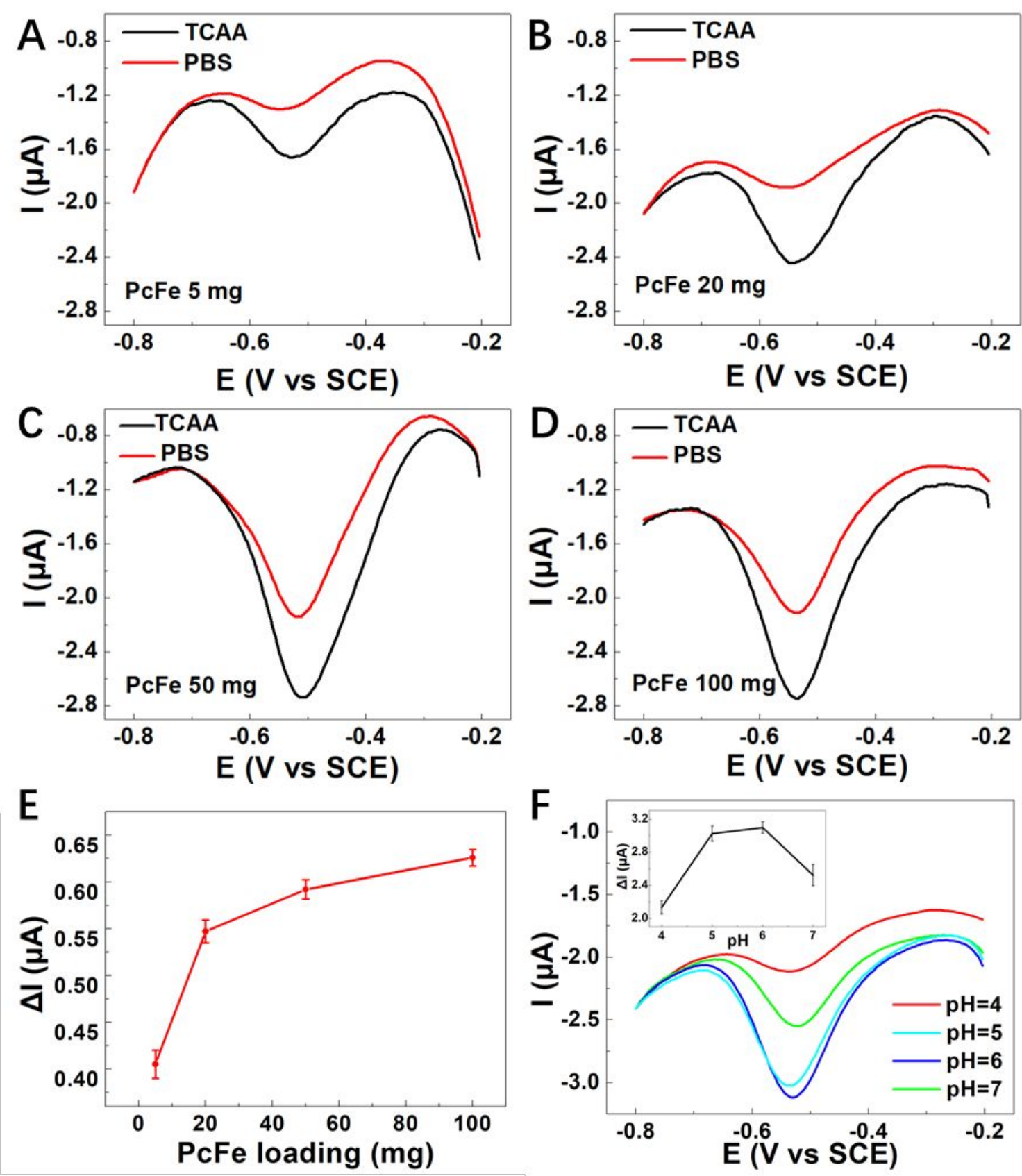

Figure S1. (A-D) Study of PcFe loading amount impact on the sensing response. (E) PcFe loading amount impact on the sensing current. (F) SWVs of the sensor tested under different $\mathrm{pH}(3,4,5$, and 6). Error bars represent the standard deviation $(n=5)$. Inset of $(F)$ is the summarized current responses under different $\mathrm{pH}$ conditions. 

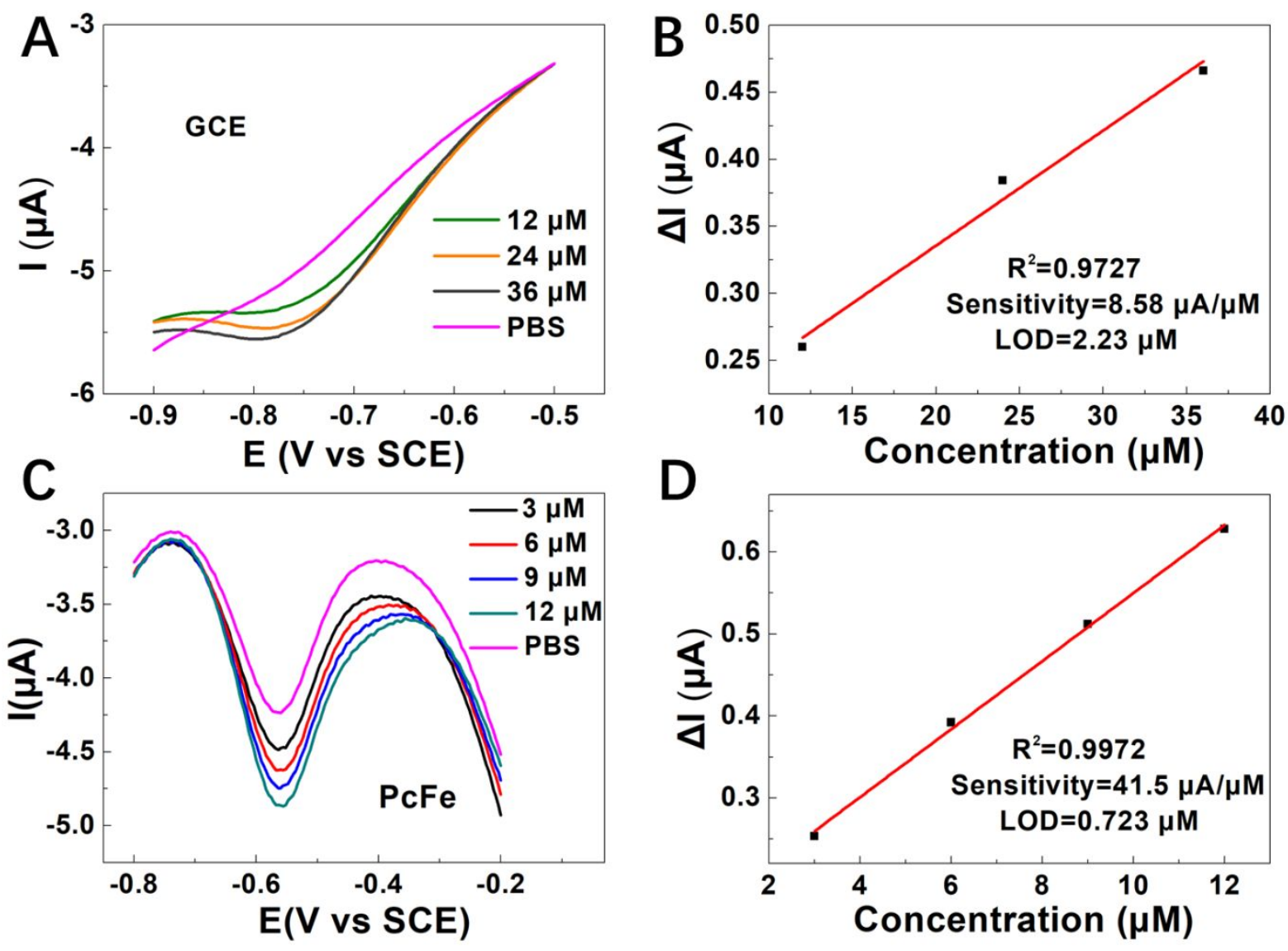

Figure S2. SWVs of (A) bare GCE and (C) PcFe electrodes to different concentrations of TCAA.

The linear relationship between $\Delta I$ and TCAA concentration of (B) bare GCE and (D) PcFe. 
Table S1 TCAA sensor performance comparison.

\begin{tabular}{|c|c|c|c|c|c|}
\hline Electrode material & Method & $\begin{array}{c}\text { Linear range } \\
(\mu \mathrm{M})\end{array}$ & $\begin{array}{l}\text { LOD } \\
(\mu \mathrm{M})\end{array}$ & $\begin{array}{l}\text { Stability } \\
\text { (\%RSD) }\end{array}$ & Reference \\
\hline $\begin{array}{l}\text { poly-NiTSPc-modifi } \\
\text { ed Au electrode }\end{array}$ & $\mathrm{CV}$ & $10-50$ & 0.6 & 85 (3 times) & 1 \\
\hline $\begin{array}{c}\mathrm{Hb} / \mathrm{Nafion} / \mathrm{GR}-\mathrm{TiO}_{2} / \\
\text { CILE }\end{array}$ & $\mathrm{CV}$ & $600-2100$ & 220 & 90.5 (30 days) & 2 \\
\hline Mb/GR-IL/CILE & $\mathrm{CV}$ & $2000-16000$ & 580 & 93.1 (3 weeks) & 3 \\
\hline $\begin{array}{c}\mathrm{Hb}-\mathrm{Ag} @ \text { GNRs-PSS- } \\
\text { PDDA }\end{array}$ & $i-t$ & $0.16-1.7$ & 0.12 & 95 (7 days) & 4 \\
\hline AgNPs-MA & SWV & $\begin{array}{l}0.1-2 \\
4-100\end{array}$ & $\begin{array}{l}0.030 \\
0.079\end{array}$ & 95 (30 days) & 5 \\
\hline Ag-MWCNT & SWV & $5-120$ & 1.9 & 93 (15 days) & 6 \\
\hline PcFe (II)@ZIF-8 & SWV & $0.02-1$ & 0.00189 & 94.7 (7 days) & This work \\
\hline
\end{tabular}

\section{Annotation:}

NiTSPc: Ni(II)-tetrasulfonated phthalocyanine; $\mathrm{Hb}$ : hemoglobin; $\mathrm{Mb}$ : myoglobin; CILE: carbon ionic liquid electrode; GR-IL: graphene-ionic liquid; GNRs: gold nanorods; PSS: polystyrene sulfonate; PDDA: poly diallyldimethylammonium chloride; AgNPs: Ag nanoparticles; MA: malic acid; MWCNT: multi-wall carbon nanotubes. 
Table S2 Recoveries of TCAA sensing for real water samples $(n=3)$.

\begin{tabular}{ccccc}
\hline Sample & $\begin{array}{c}\text { Spiked TCAA } \\
\text { concentration } \\
(\boldsymbol{\mu M})\end{array}$ & $\begin{array}{c}\text { Tested TCAA } \\
\text { concentration } \\
(\boldsymbol{\mu M})\end{array}$ & $\begin{array}{c}\text { RSD } \\
(\%)\end{array}$ & $\begin{array}{c}\text { Recovery } \\
(\mathbf{\%})\end{array}$ \\
\hline \multirow{2}{*}{ Tap water } & 0.5 & 0.466 & 0.71 & 93.2 \\
& 1 & 1.052 & 2.83 & 105.2 \\
Swimming pool water & 0.5 & 0.44 & 1.29 & 88.0 \\
& 1 & 1.031 & 3.21 & 103.1 \\
\hline
\end{tabular}

\section{References}

1. Altamar, L.; Fernandez, L.; Borras, C.; Mostany, J.; Carrero, H.; Scharifker, B. Electroreduction of chloroacetic acids (mono-, di- and tri-) at polyNi(II)-tetrasulfonated phthalocyanine gold modified electrode. Sensor. Actuat. B-Chem. 2010, 146 (1), 103-110.

2. Sun, W.; Guo, Y. Q.; Ju, X. M.; Zhang, Y. Y.; Wang, X. Z.; Sun, Z. F. Direct electrochemistry of hemoglobin on graphene and titanium dioxide nanorods composite modified electrode and its electrocatalysis. Biosens. Bioelectron. 2013, 42, 207-213.

3. Ruan, C. X.; Li, T. T.; Niu, Q. J.; Lu, M.; Lou, J.; Gao, W. M.; Sun, W. Electrochemical myoglobin biosensor based on graphene-ionic liquid-chitosan bionanocomposites: Direct electrochemistry and electrocatalysis. Electrochim. Acta 2012, 64, 183-189.

4. Qian, D. P.; Li, W. B.; Chen, F. T.; Huang, Y.; Bao, N.; Gu, H. Y.; Yu, C. M. Voltammetric sensor for trichloroacetic acid using a glassy carbon electrode modified with $\mathrm{Au} @ \mathrm{Ag}$ nanorods and hemoglobin. Microchim. Acta 2017, 184 (7), 1977-1985.

5. Bashami, R. M.; Soomro, M. T.; Khan, A. N.; Aazam, E. S.; Ismail, I. M. I.; El-Shahawi, M. S. A highly conductive thin film composite based on silver nanoparticles and malic acid for selective electrochemical sensing of trichloroacetic acid. Anal. Chim. Acta 2018, 1036, 33-48.

6. Liu, B.; Hu, X.; Deng, Y.; Yang, S.; Sun, C. Selective determination of trichloroacetic acid using silver nanoparticle coated multi-walled carbon nanotubes. Electrochem. Commun. 2010, 12 (10), 1395-1397. 